\title{
A Threat-Based Cybersecurity Risk Assessment Approach Addressing SME Needs
}

\author{
Max van Haastrecht \\ m.a.n.vanhaastrecht@uu.nl \\ Utrecht University \\ The Netherlands
}

\author{
Louis Baumgartner \\ Fachhochschule Nordwestschweiz \\ (FHNW) \\ Switzerland \\ louis.baumgartner@fhnw.ch
}

\author{
Injy Sarhan* \\ Utrecht University \\ The Netherlands \\ i.a.a.sarhan@uu.nl
}

\author{
Wissam Mallouli \\ Montimage \\ France \\ wissam.mallouli@montimage.com
}

\author{
Alireza Shojaifar \\ Fachhochschule Nordwestschweiz \\ (FHNW) \\ Switzerland \\ alireza.shojaifar@fhnw.ch
Marco Spruit ${ }^{\dagger \dagger}$
Leiden University Medical Center
(LUMC) \\ The Netherlands \\ m.r.spruit@lumc.nl
}

\begin{abstract}
Cybersecurity incidents are commonplace nowadays, and Smalland Medium-Sized Enterprises (SMEs) are exceptionally vulnerable targets. The lack of cybersecurity resources available to SMEs implies that they are less capable of dealing with cyber-attacks. Motivation to improve cybersecurity is often low, as the prerequisite knowledge and awareness to drive motivation is generally absent at SMEs. A solution that aims to help SMEs manage their cybersecurity risks should therefore not only offer a correct assessment but should also motivate SME users. From Self-Determination Theory (SDT), we know that by promoting perceived autonomy, competence, and relatedness, people can be motivated to take action. In this paper, we explain how a threat-based cybersecurity risk assessment approach can help to address the needs outlined in SDT. We propose such an approach for SMEs and outline the data requirements that facilitate automation. We present a practical application covering various user interfaces, showing how our threat-based cybersecurity risk assessment approach turns SME data into prioritised, actionable recommendations.
\end{abstract}

\section{CCS CONCEPTS}

- Security and privacy $\rightarrow$ Systems security; Human and societal aspects of security and privacy.

\section{KEYWORDS}

cybersecurity, SME, threat, risk assessment, SDT, motivation

\footnotetext{
*Also with Arab Academy for Science, Technology and Maritime Transport (AASTMT)

$\dagger$ Also with Leiden University.

¥Also with Utrecht University.

Permission to make digital or hard copies of all or part of this work for personal or classroom use is granted without fee provided that copies are not made or distributed for profit or commercial advantage and that copies bear this notice and the full citation on the first page. Copyrights for components of this work owned by others than ACM must be honored. Abstracting with credit is permitted. To copy otherwise, or republish, to post on servers or to redistribute to lists, requires prior specific permission and/or a fee. Request permissions from permissions@acm.org.

ARES 2021, August 17-20, 2021, Vienna, Austria

(C) 2021 Association for Computing Machinery.

ACM ISBN 978-1-4503-9051-4/21/08 . .\$15.00

https://doi.org/10.1145/3465481.3469199
}

ACM Reference Format:

Max van Haastrecht, Injy Sarhan, Alireza Shojaifar, Louis Baumgartner, Wissam Mallouli, and Marco Spruit. 2021. A Threat-Based Cybersecurity Risk Assessment Approach Addressing SME Needs. In The 16th International Conference on Availability, Reliability and Security (ARES 2021), August 17-20, 2021, Vienna, Austria. ACM, New York, NY, USA, 12 pages. https://doi.org/ $10.1145 / 3465481.3469199$

\section{INTRODUCTION}

Cybersecurity incidents are commonplace nowadays and can have a devastating impact on businesses [57]. Small- and Medium-Sized Enterprises (SMEs, [19]) are especially vulnerable since they have limited resources to deal with cyber-attacks [24]. Additionally, the lack of cybersecurity knowledge and awareness of SME employees causes low motivation to improve the SME cybersecurity posture [24].

A vital first step towards managing cybersecurity risks is to assess these risks [44]. Several cybersecurity risk assessment approaches tailored to SMEs exist $[33,47,56]$. From the two leading behavioural theories in the security field - Protection Motivation Theory (PMT) and Self-Determination Theory (SDT) - we know that users are most likely to take action if risk assessment solutions manage to convince the user of the risk associated with cybersecurity threats and their ability to deal with those threats [31, 32, 52]. In PMT, this translates to a focus on threat- and coping appraisal [31], whereas in SDT perceived autonomy, competence, and relatedness are seen as the main drivers of motivation.

Knowing that motivation to improve cybersecurity is relatively low among SMEs [24], it is reasonable to expect that cybersecurity risk assessment solutions for SMEs address the PMT and SDT factors. This is especially relevant for SMEs that are less digitally mature, as they are often unaware of cyber threats and require easily understandable solutions due to their limited (initial) cybersecurity knowledge [20]. Sadly, most solutions are not adapted to suit SME needs [24], with researchers insisting it is the responsibility of SMEs to take action [7, 27], rather than designing solutions that motivate SMEs $[8,46]$. By not properly addressing the psychological needs identified by PMT and SDT, these solutions are much less likely to motivate SME users [23]. 
Threat-based cybersecurity risk assessment approaches are a common tool to address the motivational issues of existing solutions. Threat-based approaches motivate threat appraisal through the incorporation of real-life threat information [22]. Additionally, as Menard et al. [32] recognise, any appeal for adopting cybersecurity countermeasures will be directly or indirectly based on a particular threat. Threat-based approaches offer a natural way to prioritise countermeasures, which is an important requirement in facilitating a usable solution for SMEs [8].

It is no surprise that threat-based approaches are common in both the privacy $[14,53]$ and cybersecurity $[3,39,54]$ fields. Threatbased cybersecurity risk assessment approaches specifically aimed at enterprises already exist $[39,50]$. However, it has been well documented that approaches for enterprises in general do not map well to the SME situation [20,24].

As a result, it is essential to discover how a threat-based cybersecurity risk assessment can be made to work for SMEs, without losing its ability to motivate users through the needs identified in PMT and SDT. This inspires the research question of this paper:

\section{How can we create a cybersecurity risk assessment approach for SMEs that promotes user motivation?}

In Section 2, we provide further insight into the context and motivation of this research. Section 3 introduces our algorithm, along with the requirements - both technically and in terms of data - for it to function properly. A practical application of our approach is outlined in Section 4. Section 5 discusses the dependencies within our solution and the privacy implications of our risk assessment approach. Finally, in Section 6, we conclude and propose ideas for future work.

\section{CONTEXT AND MOTIVATION}

The European Horizon 2020 project GEIGER [21] aims to help SMEs, and specifically micro-enterprises, to improve their cybersecurity posture and protect themselves against cybersecurity risks. The GEIGER project targets the smallest and least digitally mature SMEs. This group requires simple and understandable solutions, that nonetheless manage to address all areas of cybersecurity risk assessment [20]. We believe a threat-centric cybersecurity risk assessment approach addresses these needs.

Cybersecurity risk assessment approaches inherently include a view on threats, due to the link between the concepts of risk and threat. At times researchers make this link explicit when employing some variant of the definition risk $=$ threat $\times$ vulnerability $\times$ consequence $[11,48]$. In other approaches, such as when building on the vulnerability-threat-control paradigm [37], the link is implicit, but present.

Nevertheless, we can distinguish threat-based cybersecurity risk assessment approaches - that centrally position the threat concept from approaches that are not threat-based. In Section 2.1 we focus on cybersecurity risk assessment methodologies that are aimed at SMEs and not threat-based. These approaches will often not include the real-life threat environment [22]. Section 2.2 covers threat-based approaches not specifically geared towards SMEs.

\subsection{Cybersecurity Risk Assessment for SMEs}

Although SMEs are often addressed as a single group, in the cybersecurity context there are large differences among SMEs [20]. This motivates a need for solutions that adapt based on the organisational characteristics of SMEs, such as the SME country or region [41], the SME sector [33] and the cybersecurity knowledge available in the SME [55]. The European Digital SME Alliance additionally proposes to take into account the role that an SME plays in the digital ecosystem, distinguishing four categories: digital enablers, digitally based SMEs, digitally dependent SMEs, and start-ups [20].

To attend to the needs of SMEs, certain cybersecurity risk assessment methodologies have been adapted to be suitable for smaller businesses $[1,15]$. Maturity models are also often employed, due to their ability to provide a complete assessment while being able to adapt based on SME characteristics $[4,33,56]$. The difficulty with all of these approaches is that they generally require a certain level of cybersecurity expertise to be present at the SME and that they assume to be dealing with a motivated user. Although these assumptions may hold for digital enablers and digitally based SMEs, this certainly cannot be expected of the digitally dependent SMEs and start-ups, who generally have little to no cybersecurity knowledge and are therefore also minimally motivated to improve their cybersecurity situation [24].

Cybersecurity risk assessment solutions would be better suited to digitally dependent SMEs and start-ups if they could incorporate the important psychological factors outlined by PMT and SDT [31, 32]. Approaches explicitly incorporating behavioural theory insights are promising [46], but contain knowledge requirements that digitally dependent SMEs and start-ups cannot fulfil. Threatbased risk assessment approaches offer interesting possibilities to assist these least digitally mature SMEs.

\subsection{Threat-Based Cybersecurity Risk Assessment}

Threat-based cybersecurity risk assessment approaches are not commonly applied to SMEs. That certainly does not imply, however, that these approaches are not prominent. In privacy risk assessment, the ability to prioritise controls from a threat-based methodology is one of the reasons mentioned for preferring such an approach [14]. In cybersecurity risk assessment, threat-based approaches are popular not only for their prioritisation ability [3, 34, 39], but also due to their ability to facilitate automation through threat catalogues [9] and publicly shared incident information [29]. Common risk assessment methodologies used in practice, such as STRIDE [42] and OCTAVE [50], are also regularly threat-based.

The prevalence of threat-based cybersecurity risk assessment methodologies aligns with the observation that real-life threat information should be incorporated in these approaches [22]. Threat appraisal is central in PMT and surfaces when applying SDT in the cybersecurity setting $[32,36]$. By using insights from PMT and SDT to design appropriate nudges $[46,52]$, threat-based approaches have the potential to be highly suitable to SMEs [28].

We can conclude that threat-based cybersecurity risk assessment approaches can motivate SMEs to improve their cybersecurity under the right circumstances. The least digitally mature SMEs digitally dependent SMEs and start-ups - stand to gain the most 
[20]. Nevertheless, threat-based approaches are not commonly employed to assist SMEs. In the remainder of this paper, we formulate a threat-based cybersecurity risk assessment approach for SMEs and argue for the motivational benefits of such an approach.

\section{A THREAT-BASED CYBERSECURITY RISK INDICATOR}

A threat-based cybersecurity risk assessment algorithm must be supported by a data model and data sources that are equally threatcentric. In this section, we describe how a threat-based view of SME cyber-systems produces a data model supporting a threat-based approach to cybersecurity risk assessment. We outline the data required to enable our approach and describe the algorithm that transforms the data into a cybersecurity risk indicator.

\subsection{Data Model}

The impetus for an SME owner to perform a cybersecurity risk assessment is that they want to learn how to protect their SME. Figure 1, adapted from Casola et al. [10], shows how this original motivation serves as one of the aspects involved in a threat-based cybersecurity risk assessment. The SME consists of assets that are valuable to the SME, such as users and devices.

The vulnerability-threat-control paradigm [37] is a general framework that can be used as a basis for our assessment approach. Within the paradigm assets can have vulnerabilities that can be exploited by threats, leading to loss or harm. Cybersecurity metrics can be used to indicate the cybersecurity risk faced by a particular asset. Cybersecurity metrics result from measuring the cybersecurity properties of an asset. The metric value should correlate to the vulnerability of the asset being measured so that it can be used in assessing risk. In this context, the risk indication given by cybersecurity metrics signifies the potential of threats to exploit vulnerabilities. To counter vulnerabilities and mitigate risk, the SME owner can enforce countermeasures, which are sometimes referred to as controls.

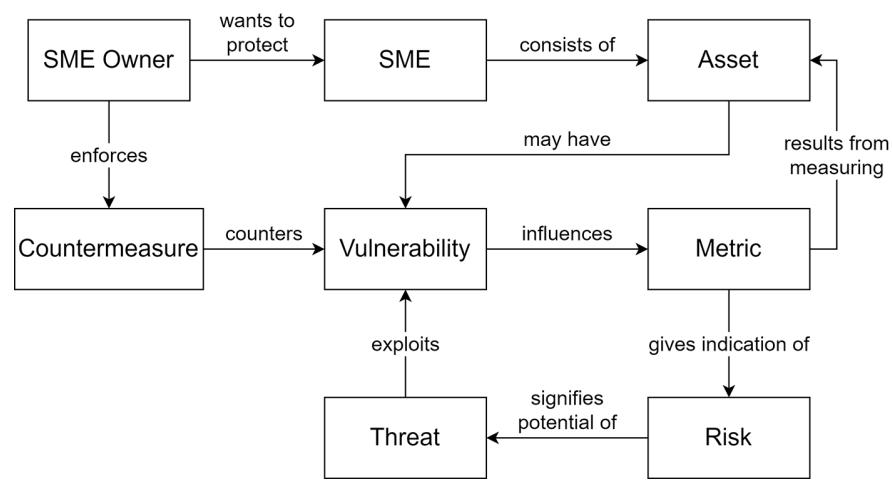

Figure 1: View on cyber-systems, adapted from Casola et al. [10] to fit a threat-based cybersecurity risk assessment approach for SMEs.

Although the model in Figure 1 provides a clear depiction of the concepts involved in our threat-based approach, it is not detailed enough to serve as a basis for defining our algorithm data requirements. Figure 2, a conceptual data model, addresses this issue.

The risk profile, location, and sector elements of the enterprise entity shown in Figure 2 allow the algorithm to adapt based on the characteristics of the SME. Threats, metrics, and recommendations are core elements of our model. We use the term recommendation rather than countermeasure within the GEIGER solution, to distinguish the textual explanation and motivation (recommendation) which is the element shown to the user of our application - from the action it describes (countermeasure). Both the recommendations and metrics of our solution are related to threats, which have a central position in our approach.

The metrics of our GEIGER solution measure two types of assets: users and devices. For users, we measure their knowledge and ability through interactive cybersecurity training and education. Device metrics result from the measurement of device properties by tools incorporated in the GEIGER solution. The metric values we calculate allow us to determine an indication of the cybersecurity risk faced by the SME: the GEIGER score. We can then present the user with the most relevant recommendations, where relevance is determined by the impact that the countermeasures corresponding to the recommendations have on the threats included in the GEIGER solution. The user can implement countermeasures based on the suggested recommendations, to counter vulnerabilities and mitigate risk. Implemented countermeasures lead to an improved GEIGER score.

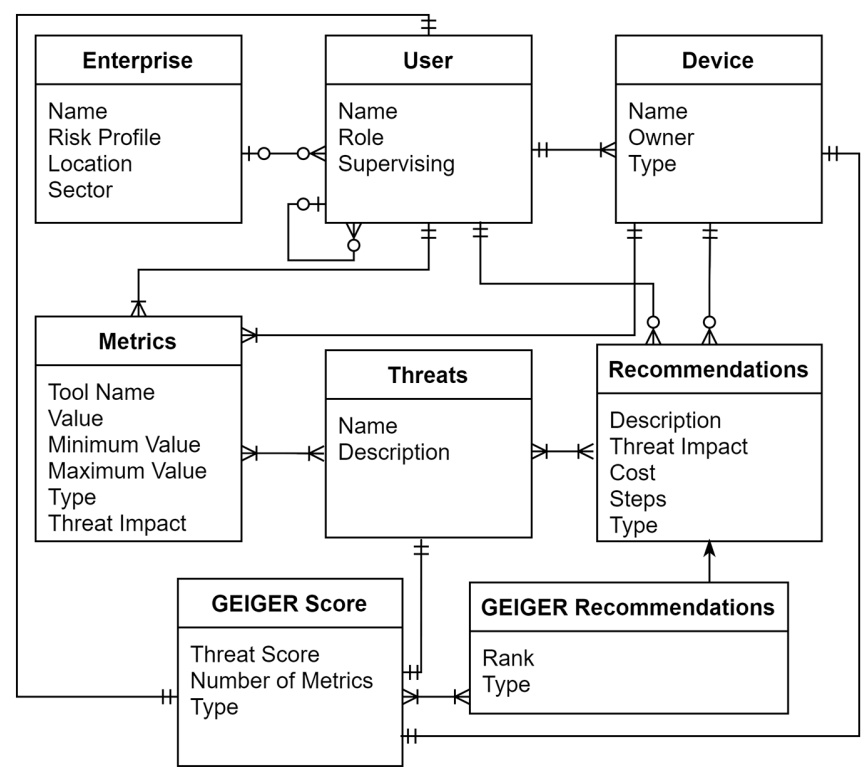

Figure 2: The conceptual data model underlying our threatbased cybersecurity risk assessment approach.

\subsection{Data Requirements}

From Figure 2 we can derive the three main inputs required for our algorithm: metrics, threats, and recommendations. Each metric and each recommendation must relate to at least one threat. 
Additionally, as discussed in Section 2.1, our algorithm must be able to adapt to different SME profiles. For the GEIGER project, we focus on three specific characteristics to form the SME profile: the SME category [20], the SME country, and the SME sector. The required data then enters the system as global algorithm settings through the curator of the project, as aggregate data from Computer Emergency Response Teams (CERTs) linked to the solution, through the user entering data, or from tools that are linked to the solution. This process is depicted in Figure 3.

Figure 3 shows how users interact with the local component and how CERTs and the curator provide data to the cloud component of the solution. The local component is the application the user installs on their device. The cloud component is required to facilitate data sharing, as well as to update the algorithm based on new insights and data.

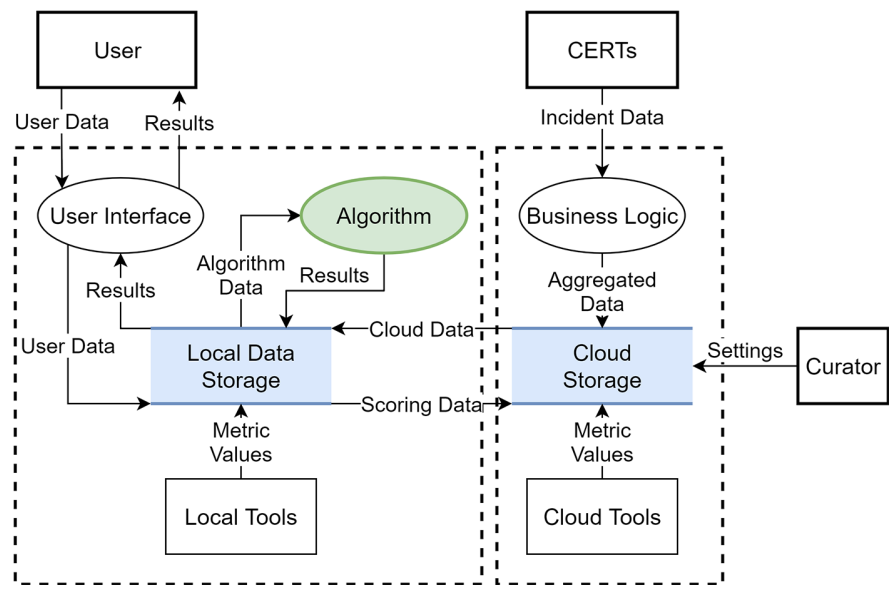

Figure 3: Data flow diagram showing how data from various sources flows through the system to be used in the algorithm.

To define the threats that should be considered for our SME target group, we look towards the European Union Agency for Cybersecurity (ENISA). Since 2012, ENISA publishes an annual list of top cybersecurity threats [30]. Through the years the list has remained remarkably unchanged, which is why it serves as an excellent basis for our threat-based approach. From the list of top threats in 2020 [18], we select those threats which have been present since the first list in 2012 and are not indicated by ENISA to be part of another threat [17]. An exception is ransomware, which is a type of malware, but is considered to be a sufficiently significant threat to SMEs on its own to warrant inclusion.

To this set of threats, we add a threat category covering legal, third party, and supply chain threats. These three threats are a part of the general ENISA taxonomy [16]. They are especially relevant to our SME target group, who have a large dependency on third parties in the digital environment $[20,24]$. We name this category 'external environment threats', using terminology from socio-technical systems [12]. This gives the following threats, in order of appearance of the ENISA top threats:

- Malware,
- Web-based threats,

- Phishing,

- Web application threats,

- Spam,

- Denial of service,

- Data breach,

- Insider threats,

- Botnets,

- Physical threats,

- Ransomware,

- External environment threats.

Figure 1 shows that metrics result from measuring the properties of assets within the SME. Assets in our solution are classified as employees or devices. The properties of these assets can either be measured directly, or employees of the SME can be asked to provide the necessary information on the assets. Within the GEIGER solution, we choose to (mainly) source our data from the direct measurement of asset properties by tools included within the solution. This is shown in Figure 3, by the data flows from local and cloud tools to their respective data storages.

Besides improving metric values, SMEs can also implement countermeasures (or controls) to counter vulnerabilities. Common countermeasures can be sourced from a variety of parties, from National Cyber Security Centres (NCSCs) and CERTs [35, 49], to standards organisations [25, 26], to peer-reviewed research [57]. In our SME context, we should be able to argue that the countermeasures included in our solution are both necessary and sufficient. We should not include more countermeasures than necessary, to keep our solution simple. At the same time, the countermeasures we include should be sufficient to cover all relevant areas of cybersecurity.

To address this issue we followed the following process. We first collected a large set of over 300 countermeasures from publicly available sources. We distilled this list to remove duplicates. We then mapped our list to a standard set of security countermeasure categories [26], to see which countermeasures could be removed without losing coverage of a category. This process left a set of necessary and sufficient countermeasures, of which four examples are shown in Table 1.

For a functioning threat-based cybersecurity risk assessment approach, we do not only need to define the necessary components, but we also need to determine their relationships. In our concept, both metrics and countermeasures impact threats. Furthermore, each metric and countermeasure impacts only a subset of all threats. Once tool owners and the curator of the solution have established which metrics and countermeasures relate to which threats, they must then determine impacts. To guide this process, we base ourselves on the NIST Cybersecurity Framework [5], which has been used to guide cybersecurity evaluation for SMEs before [7].

The NIST framework distinguishes five core functions: identify, protect, detect, respond, and recover. The functions can be related to various stages of a cybersecurity incident, from before the incident (identify, protect), to during the incident (detect, respond), to after the incident (recover). Since each phase is increasingly less likely to occur, the impact of countermeasures and metrics in these phases also decreases. Our approach, therefore, defines a default impact of 'high' for countermeasures and metrics relating to the identify 
and protect functions, 'medium' for those relating to the detect and respond function, and 'low' for those relating to the recover function.

Table 1: An indication of the impact of metrics and countermeasures on the common SME cybersecurity threats of phishing and malware. Green arrows indicate improving scores, whereas red arrows indicate that scores worsen.

\begin{tabular}{lll} 
Metric & Threat & Countermeasure \\
\hline Malicious URLs count $\longrightarrow$ Phishing & Money transfer policy \\
Awareness training score & & Limit access \\
Malware infection count & Malware & Employ mail filtering \\
Malicious app count & & Update regularly
\end{tabular}

The final piece of the puzzle, that allows us to calculate a single indicator value for an SME, is determining the relative risks associated with each threat for each SME profile. This involves making estimates of impacts and likelihoods, to calculate the common risk value: risk $=$ impact $\times$ likelihood $[48,50]$. By surveying experts as well as security literature and reports, we can gain initial insights. However, this will not be sufficient to formulate risk estimations for each SME profile, which is an essential part of creating an adaptable approach [4].

This is why we propose to use CERT incident data to be able to create risk estimations per profile. Figure 3 shows how CERT incident data can be fed into our solution and aggregated, to then be used in determining threat-specific risks for each SME profile. Besides facilitating adaptability, the CERT incident data also allows us to incorporate real-life threat information into our solution. We hope this will promote perceived relatedness among SMEs.

\subsection{Algorithm Description}

In this section, we will describe the general mathematical representation of our algorithm. An SME can be seen as a cyber-system using the definition of Refsdal et al. [38]. Similarly, each asset of the SME, such as an employee or device, can be seen as a cyber-system. This allows us to formulate an algorithm that assesses sub-systems and recursively iterates to arrive at an overall SME score.

Let $S$ be the total set of cyber-systems of the SME, including the SME itself. Let $T$ be the set of threats and $P$ the set of SME profiles. Each combination of threat $t \in T$ and profile $p \in P$ has an associated relative risk $r_{p t} \in(0,100]$.

Let $M$ be the set of metrics. The normalised value of a metric $m \in$ $M$ for cyber-system $s \in S$ is given by $v_{m s} \in[0,1]$. We distinguish metrics that indicate improved security from metrics that indicate worsened security. Theoretically, a single metric may even relate positively to security for one threat, but negatively for another. Hence, we define the Boolean indicator $\delta_{m t}$, which equals 1 when a metric $m \in M$ relates positively to the relative risk associated with threat $t \in T$.

We further define the impact of metric $m \in M$ on threat $t \in T$ as $i_{m t}$. Recall that this impact may either be low, medium or high. We map these categories to values of $0.1,0.5$, and 1.0 , respectively. To be able to keep track of which metrics have been calculated, we define the Boolean variable $\lambda_{m s}$, which equals 1 if metric $m \in M$ has been calculated for cyber-system $s \in S$.
We let $C$ be the set of countermeasures. The variable $i_{c t}$ has an identical definition as in the metric case. The Boolean variable $\lambda_{c s}$ is now used to indicate whether a countermeasure $c \in C$ has been implemented for cyber-system $s \in S$. Since we only allow for countermeasures to be implemented or not implemented, without assigning a specific value, we have no analogue for the variable $v_{m s}$ specifying the metric value. Similarly, since countermeasures always relate positively to security, there is no analogue to the $\delta_{m t}$ variable.

All of our defined variables allow us to calculate the indicator value $I_{s p t}$ specific to threat $t \in T$, for a cyber-system $s \in S$, which is (part of) an SME with profile $p \in P$ :

$$
\begin{aligned}
I_{s p t}=50 & +50 \cdot \frac{\sum_{m \in M} \delta_{m t} \cdot \lambda_{m s} \cdot i_{m t} \cdot v_{m s}}{\sum_{m \in M} \delta_{m t} \cdot \lambda_{m s} \cdot i_{m t}} \\
& -25 \cdot\left(\frac{\sum_{m \in M}\left(1-\delta_{m t}\right) \cdot \lambda_{m s} \cdot i_{m t} \cdot v_{m s}}{\sum_{m \in M}\left(1-\delta_{m t}\right) \cdot \lambda_{m s} \cdot i_{m t}}+\frac{\sum_{c \in C} \lambda_{c s} \cdot i_{c t}}{\sum_{c \in C} i_{c t}}\right) .
\end{aligned}
$$

Equation 1 ensures the indicator value $I_{s p t}$ ranges from 0 to 100 and initially takes a value of 50 . Note that our current assumption is that countermeasures always apply to all cyber-systems under consideration. However, if necessary, the algorithm could easily be extended with an additional Boolean variable to permit variation in this dimension.

Some of the divisors of Equation 1 equal 0 when no values have been calculated. In this scenario, we set the value of the relevant fraction to 0 . The total indicator score over all threats, again ranging between 0 and 100, is given by:

$$
I_{s p}=\frac{\sum_{t \in T} I_{s p t} \cdot r_{p t}}{\sum_{t \in T} r_{p t}} .
$$

In essence, Equation 2 could be used to calculate the indicator value for the complete SME, if the system $s \in S$ considered is the SME itself. However, in practice, there are privacy constraints to sharing all data within the full company. Some of this data, especially the security information related to employees, can be sensitive. So, we need to formulate a process to arrive at an indicator value representing the entire SME, without needing to share all data items.

To solve this issue we recognise that SMEs, like any enterprise, are generally hierarchically structured. The owner of the SME is positioned at the top of the hierarchy and supervises one or more employees. These employees, in turn, may supervise further employees. By incorporating this supervision structure in our scoring mechanism, we can ensure that a minimal amount of data is shared, while still arriving at an indicator value that accurately represents the complete SME.

Within our approach, we distinguish two types of scores: user scores and device scores. User scores relate to the knowledge and ability of an employee within the SME, whereas device scores relate to the security properties of the device. Each employee $e \in S$ that has installed the GEIGER application on a device they own will therefore have at least two scores: their user score and the score of the device they own. An employee may own multiple devices and can therefore have more than two associated scores. 
An employee may not wish to share their user score per threat with their supervisor, due to the sensitive nature of this information. This is why we propose to only share aggregated data. Let $n_{s}$ be the total number of metrics calculated to arrive at the indicator value for cyber-system $s \in S$. Based on our earlier definitions, we have:

$$
n_{s}=\sum_{m \in M} \lambda_{m s}
$$

We define the set of employees $E \subset S$, to help in addressing supervision. We then define $S_{e} \subseteq S$ to be the set of cyber-systems belonging to employee $e \in E$. This set corresponds to the employee themselves and the devices they own. Let $E_{e} \subset E$ be the set of employees supervised by employee $e \in E$. We then define the aggregate score of employee $e \in E$ as:

$$
I_{e p}^{a g g}=\frac{\sum_{s \in S_{e}} I_{s p} \cdot n_{s}+\sum_{\hat{e} \in E_{e}} I_{\hat{e} p}^{a g g} \cdot n_{\hat{e}}^{a g g}}{\sum_{s \in S_{e}} n_{s}+\sum_{\hat{e} \in E_{e}} n_{\hat{e}}^{a g g}},
$$

where:

$$
n_{e}^{a g g}=\sum_{s \in S_{e}} n_{s}+\sum_{\hat{e} \in E_{e}} n_{\hat{e}}^{a g g} .
$$

The recursive nature of Equation 3 and Equation 4 allow us to iteratively calculate aggregate scores until we reach the aggregate score of the SME owner. The aggregate score of the SME owner represents all of the information available for scoring, and therefore accurately represents the cybersecurity posture of the SME. Since only aggregate data is shared, the scoring procedure preserves privacy while still managing to achieve an accurate score. Table 2 provides an overview of all of the variables discussed in this section.

The formulation of our algorithm allows us to determine the place our threat-based cybersecurity risk assessment approach takes within the information security risk assessment (ISRA) taxonomy of Shameli-Sendi et al. [44]. Our approach is quantitative and asset-driven. Additionally, assets are evaluated independently of each other and risk assessment scores are propagated through recursive formulas. Furthermore, we do not assign a monetary value to assets.

Based on the ISRA taxonomy, our approach is similar to other risk assessment approaches [2, 6, 43]. However, none of these methodologies uses threat-based techniques, nor do they use the hierarchical structure we propose to use for SMEs. We can conclude that although our approach follows established guidelines for formulating a cybersecurity risk assessment methodology, it has unique elements. These elements are included to make our approach suitable for SMEs. The following section provides further explanation on how our algorithm results are translated into visual representations to effectively nudge SME users.

\section{EXEMPLAR OF PRACTICAL APPLICATION}

Self-Determination Theory (SDT) is a theoretical framework used in the study of motivational dynamics and individual behaviours $[13,40]$. SDT distinguishes intrinsic and extrinsic types of motivation and explains people's psychology of being self-determined to adopt behaviour and persist in an activity. SDT elaborates three fundamental psychological needs - autonomy, competence, and relatedness - and assumes that their satisfaction leads to self-motivation, engagement, and positive outcomes [51].
- Autonomy: A desire to engage in activities with willingness and a freedom of choice,

- Competence: A desire to interact effectively with the environment for developing wanted outcomes and preventing undesired events,

- Relatedness: A sense of belongingness and connectedness to others or a social environment.

SDT is applied in cybersecurity [32] and security solution design $[45,46]$ to explain the relationships between design features and user motivation in cybersecurity. The basic psychological needs are reliable mediators to study how security tool features support user need satisfaction and consequent tool adoption. This section presents the main GEIGER toolbox interfaces and outlines how the toolbox features operationalised SDT constructs (autonomy, competence, and relatedness) to encourage users to adopt GEIGER for protecting their companies.

\subsection{Main Interface}

The structure of the main screen depicted in Figure 4 follows the approach that the most important elements are displayed on top. If a risk scan has already been carried out, the first thing the user sees is their aggregated score, which is displayed in green (low), yellow (medium), orange (high), or red (very high), depending on the level of the risk. This gives a first impression of the overall risk potential and should trigger the need to act depending on the threat situation.

The score is shown noticeably large because it is an aggregation of the user scores and the device scores across all threats. Depending on the role of the user, the labelling of the score adapts to convey whether the score represents the whole company or just one person with its employees. The aggregated score and its colour support the user's familiarity with the overall potential risks in the company and motivate the user for a desirable practice.

By pressing the scan risk button, the calculation of the latest risk score is initiated. An intermediate screen shows that the app is working in the background and how far advanced the calculation process is. Furthermore, during this waiting period, the user should be shown how their aggregated score is achieved, as well as those of the employees they supervise. As soon as the calculation process has finished, the main screen will be shown again with the current aggregated score of the user as well as all threats with their current scores.

Threats with higher risk scores are shown first. Each threat is shown as a so-called card with the threat name, a threat visualisation, a threat score, and a button that leads to the recommendations for a threat. The button 'improve' is coloured green, which contrasts with the colours of high-risk scores to convey a positive action.

To get a quick overview of the situation of other devices or employees, the coloured dots below the buttons show how many devices or employees have been classified with which risk level (left image of Figure 4).

\subsection{Device and Employee Risk}

Using the buttons 'device risks' and 'employee risks' of Figure 4, the user can either navigate to a list with all their devices or to a list with all their employees. Here, the aggregated scores over all threats 
Table 2: The variables used within the algorithm.

\begin{tabular}{ll} 
Variable & Definition \\
\hline$S$ & The set of all cyber-systems within the SME. \\
$S_{e}$ & The set of cyber-systems belonging to employee $e \in E, S_{e} \subseteq S$. \\
$E$ & The set of all employees within the SME, $E \subset S$. \\
$E_{e}$ & The set of employees supervised by employee $e \in E, E_{e} \subset E$. \\
$T$ & The set of all threats. \\
$P$ & The set of all SME profiles. \\
$M$ & The set of all metrics. \\
$C$ & The set of all countermeasures. \\
$r_{p t}$ & Relative risk of threat $t \in T$, for profile $p \in P$. \\
$v_{m s}$ & Normalised value of metric $m \in M$, for cyber-system $s \in S$. \\
$i_{m t}$ & Impact of metric $m \in M$ on threat $t \in T$. \\
$i_{c t}$ & Impact of countermeasure $c \in C$ on threat $t \in T$. \\
$\lambda_{m s}$ & Boolean variable equalling 1 when metric $m \in M$ has been calculated for cyber-system $s \in S$. \\
$\lambda_{c s}$ & Boolean variable equalling 1 when countermeasure $c \in C$ is implemented for cyber-system $s \in S$. \\
$\delta_{m t}$ & Boolean variable equalling 1 when metric $m \in M$ relates positively to the risk of threat $t \in T$. \\
$I_{s p t}$ & Threat-specific cybersecurity risk indicator for cyber-system $s \in S$. \\
$I_{s p}$ & Cybersecurity risk indicator for cyber-system $s \in S$. \\
$I_{e p}^{a g g}$ & Aggregate cybersecurity risk indicator for employee $e \in E$. \\
$n_{e}^{a g g}$ & Total number of metrics calculated to arrive at $I_{e p}^{a g g}$.
\end{tabular}

are displayed for each device or employee (Figure 5). The employee and device lists help the user to better handle security measures in the company. Moreover, the prioritised list of visualised threats and texts and the available tailored recommendations support user competence and autonomy.

In general, as soon as a scan is carried out, the scores of the devices are no longer up to date. This is depicted in the device risk screen of Figure 5. The device is marked and the user is prompted to open the app on the device and perform a scan.

In the case of employees, when the supervisor scans, they receive a request to allow or deny sharing their scores with their supervisor. For this reason, either the score is displayed on the employee screen if permission has been granted, or the score is displayed as pending or rejected (right image of Figure 5). Information sharing in GEIGER is based on users' permission. A user may choose to allow or deny sharing their information with the supervisor, stimulating perceived autonomy.

\subsection{Recommendations}

Using a tab, the user can switch between user- and device-specific recommendations and sees the respective score directly on the tab (left image of Figure 6). Depending on the tab, the user is shown either their name or that of their active device.

Since the target group may still be unfamiliar with threat terminology or with the concept of user and device scores, they are given the opportunity to obtain additional information. Figure 6 shows how this information can be accessed, for example, via a button labelled 'About Phishing' or 'About User Score.' To prevent flooding the user with information, the respective input is presented in the form of several small blocks and with corresponding illustrations.

The recommendations with the highest impact on risk reduction are displayed, given that they correspond to the knowledge level of the user and are yet to be implemented. Recommendations that have been implemented are marked with a green tick. Each recommendation is categorised with a risk reduction impact of low, medium, or high.

The recommendations can contain learning content so that the user is more likely to recognise dangers and improve their behaviour in the long term. There are also recommendations in which the user must implement a precautionary measure, guided by step-bystep instructions. The user can implement some recommendations directly with the help of the app, while others require additional tools that take on more complex tasks.

Recommendations that could be too demanding are marked as 'Difficult,' whereby the user is asked to contact a security defender if necessary. In any case, the user can use the 'Get Help' button to access a list of security defenders to receive more personal support. The recommendation support embedded in GEIGER helps to promote perceived autonomy and competence. By enabling contact with a trusted advisor, in the form of a security defender, we hope to stimulate perceived relatedness and competence among users.

The GEIGER features are designed to provide information and familiarity with different types of potential security threats and improve user experience. Various colours and scores support users' appraisal of the risks, and in turn, support extrinsic motivation to enact security measures [36]. Consistent with SDT's three basic 


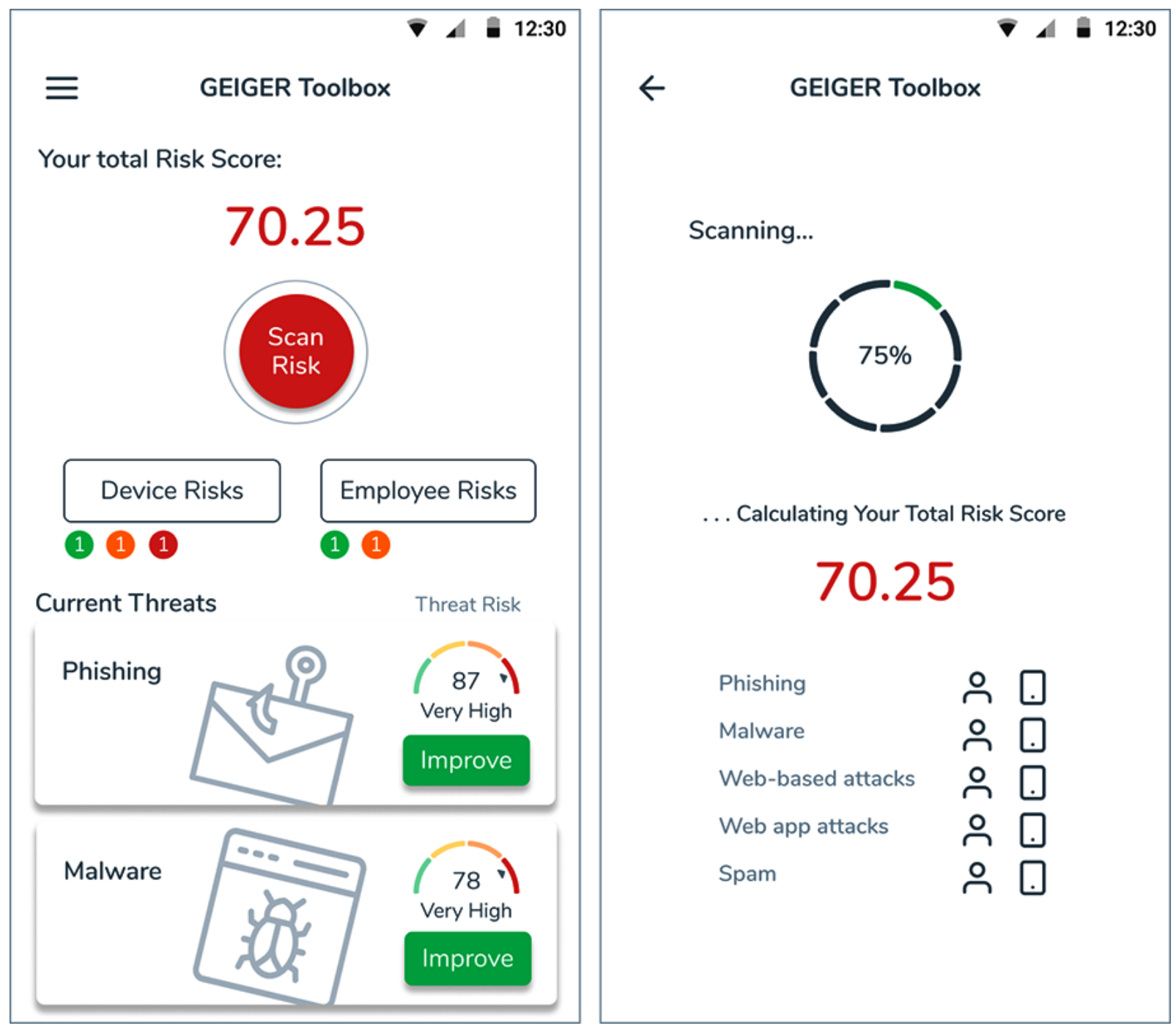

Figure 4: Main interface (left) and score calculation process (right).

psychological needs, GEIGER features are designed to facilitate daily self-determined cybersecurity improvement.

\section{DISCUSSION AND LIMITATIONS}

The GEIGER indicator relies on several threat-related metrics collected by different GEIGER tools to provide relevant insight into the risk level of an SME, including its devices and employees. This module is part of the GEIGER ecosystem composed of scanning tools (for threat detection), education tools (for training) and components integrating data coming from different CERTs.

The confidence in the GEIGER indicator depends on the completeness of the collected data. In other words, the more data that is available and recent, the more accurate the GEIGER indicator is. Ideally, the uncertainty associated with a lack of data would be quantified and communicated to the user. Although this is currently not part of the GEIGER user interface, it could prove to be a valuable addition.
The GEIGER solution is composed of several interdependent components. The accuracy of the GEIGER indicator may come at a cost; the cost of complexity. We should take care to translate this underlying complexity into a simple and clear message to the user, which is what we aim to achieve with the user interface outlined in Section 4.

An important facet in harbouring user trust is adequately addressing confidentiality concerns [45]. The GEIGER indicator is computed for each employee and no sharing - to the employees' supervisor or the GEIGER cloud - is allowed before the consent of this employee. The GEIGER indicator is GDPR-compliant by respecting user preferences regarding data privacy.

Yet, we wish to go further than just compliance. Since the accuracy of the GEIGER indicator is largely determined by the amount of data underlying its value, it will be necessary to create a comfortable environment for the user to provide consent to information sharing [45]. However, we recognise that it will be challenging to 


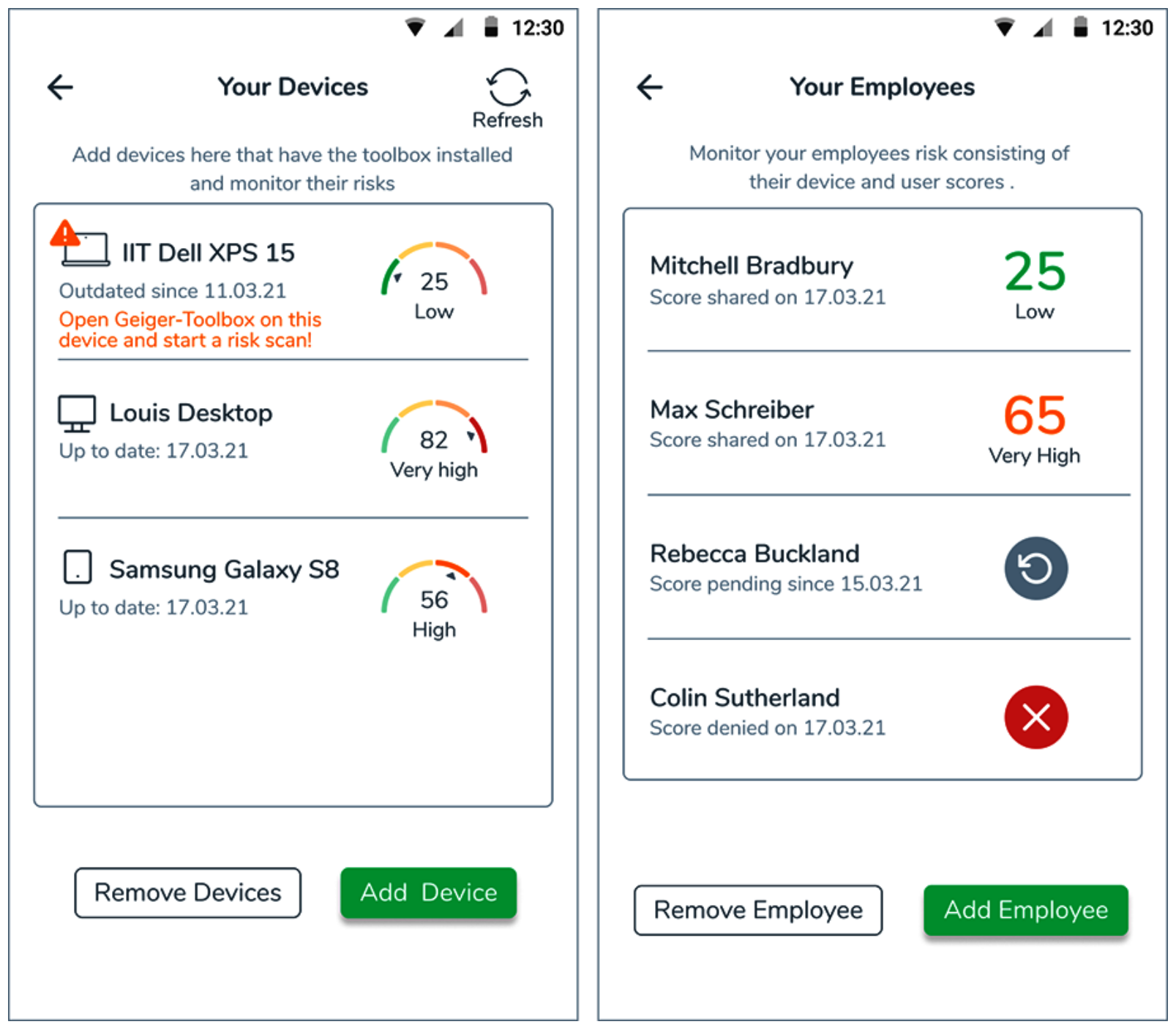

Figure 5: Interfaces of all devices (left) and all employees (right) with respective risk scores.

find the right balance between pushing users to share data and providing a comfortable setting, as these are somewhat conflicting goals.

The GEIGER indicator is still in its prototype release. More validation with end-user SMEs is planned in the coming months to refine its scope and improve its reliability in terms of the suggested recommendations to protect SMEs from the most impactful cyberthreats.

\section{CONCLUSION AND FUTURE WORK}

Less digitally mature Small- and Medium-Sized Enterprises (SMEs) are perhaps the most vulnerable to cybersecurity threats of all organisations. These SMEs often lack the cybersecurity knowledge, awareness, and resources to deal with cyber-attacks. Perhaps even more worryingly, their limited connection to the cybersecurity topic often causes a low motivation to improve their cybersecurity posture. This is why we set out to answer the question: How can we create a cybersecurity risk assessment approach for SMEs that promotes user motivation?

Any appeal for adopting cybersecurity countermeasures is, directly or indirectly, motivated by a particular threat. Unsurprisingly, threat-based cybersecurity risk assessment methodologies are a popular tool. Besides having a natural ability to promote threat appraisal, an important concept in behavioural theories such as Protection Motivation Theory (PMT) and Self-Determination Theory (SDT), threat-based approaches facilitate automation and prioritisation.

Nevertheless, threat-based cybersecurity risk assessment approaches are not commonly used to assist SMEs. We introduced a threat-based cybersecurity risk indicator specifically aimed at SMEs and discussed the data requirements to make the algorithm behind such an indicator work. After outlining the details of our algorithm, we covered a practical application of our approach, delineating 


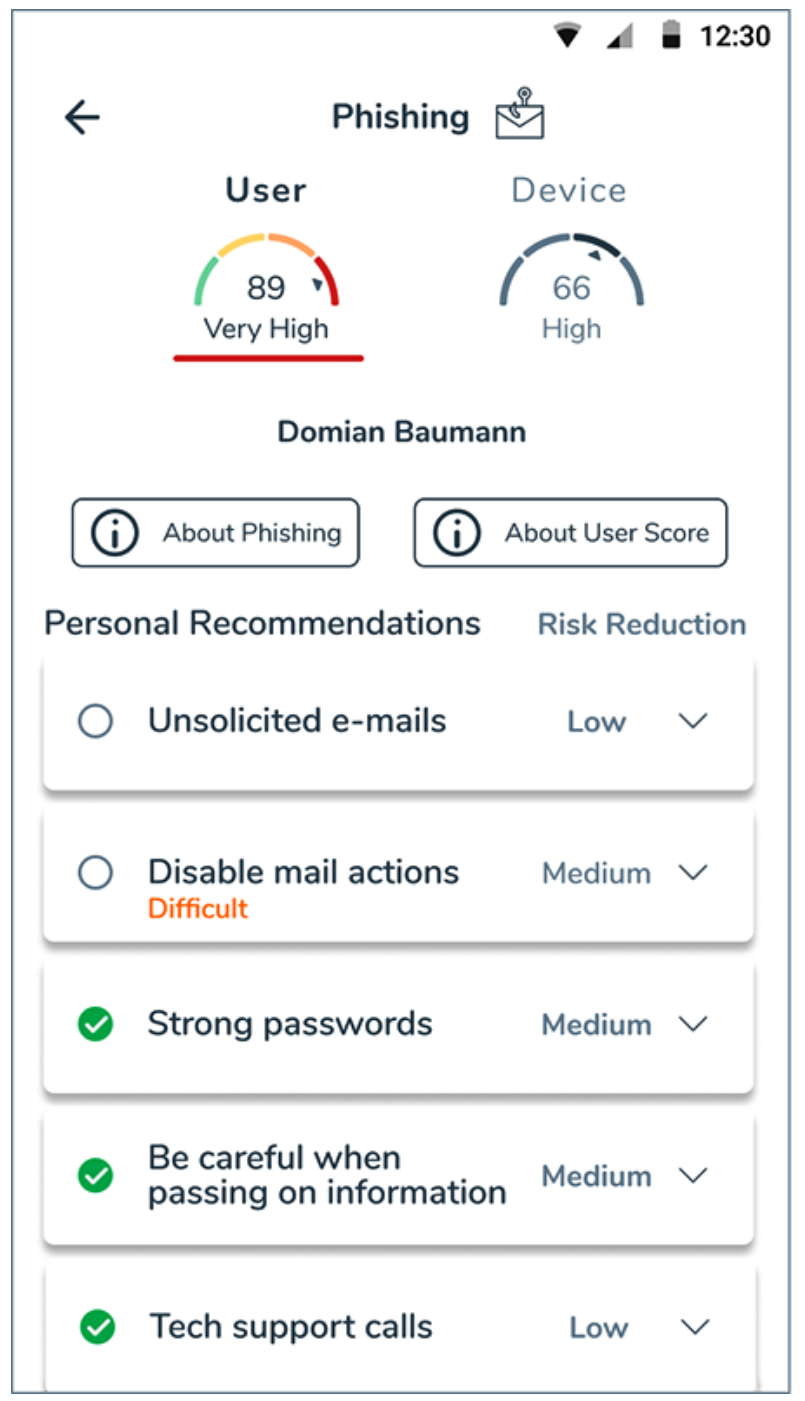

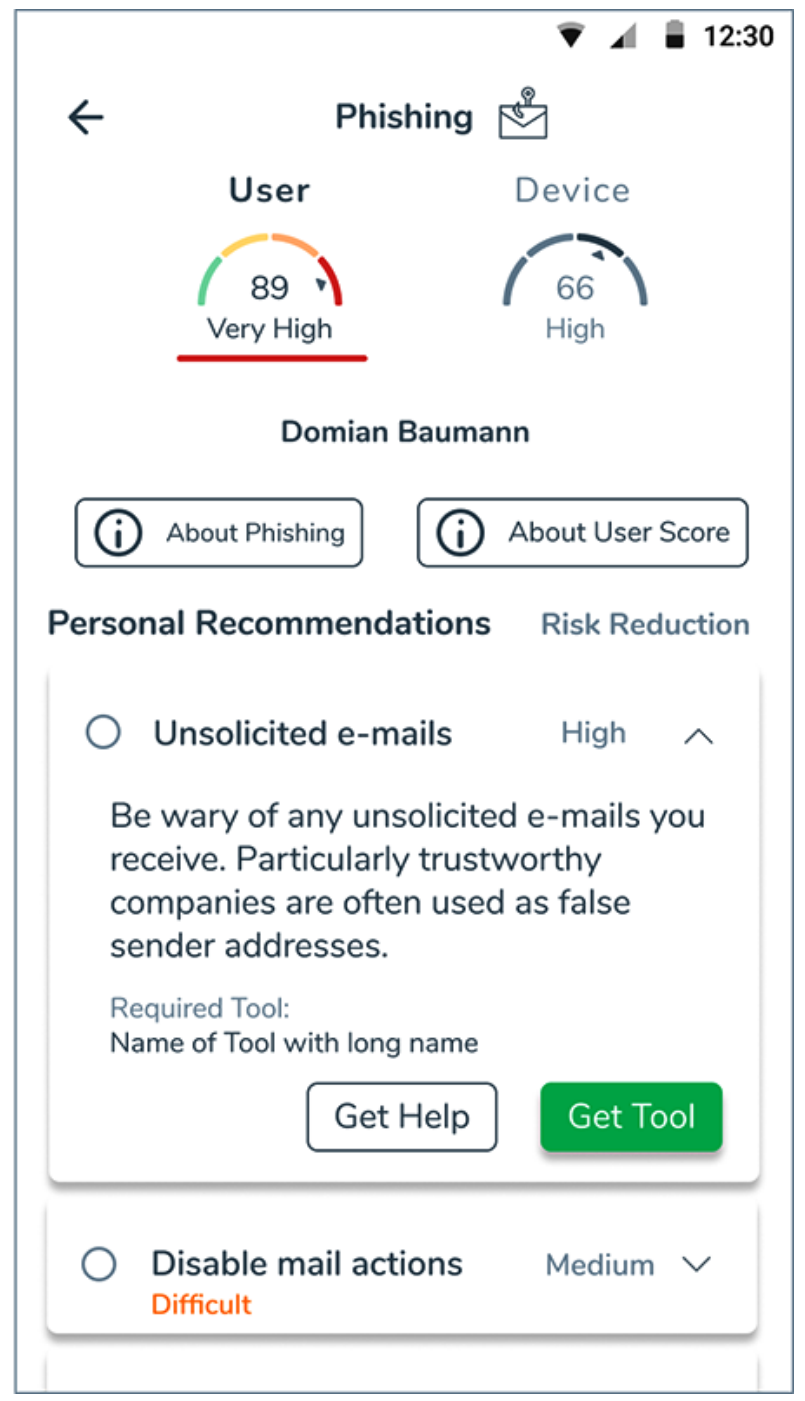

Figure 6: Interfaces of user-specific recommendations for phishing.

how different user interface screens satisfied the three SDT needs: autonomy, competence, and relatedness.

Our work shows that it is feasible to create a cybersecurity risk assessment approach for SMEs that promotes user motivation. We strongly believe that threats should play a central role in any such solution.

We recognise that challenges remain and that more validation of our approach is necessary. In future work, we plan to refine our algorithm through the incorporation of extensive user feedback. Additionally, we intend to further investigate threat prioritisation and the possibilities of incorporating privacy-preserving ideas in our algorithm. We hope that the new insights we gain will bring the most vulnerable SMEs another step closer to security.

\section{ACKNOWLEDGMENTS}

This work was made possible with funding from the European Union's Horizon 2020 research and innovation programme, under grant agreement No. 883588 (GEIGER). The opinions expressed and arguments employed herein do not necessarily reflect the official views of the funding body.

\section{REFERENCES}

[1] Christopher J. Alberts, Audrey J. Dorofee, James F. Stevens, and Carol Woody. 2005. OCTAVE-S Implementation Guide, Version 1. Technical Report. Software Engineering Institute, Carnegie Mellon University.

[2] Tansu Alpcan and Nick Bambos. 2009. Modeling Dependencies in Security Risk Management. In 2009 Fourth International Conference on Risks and Security of Internet and Systems (CRiSIS 2009). 113-116. https://doi.org/10.1109/CRISIS.2009. 5411969

[3] A. W. Atamli and A. Martin. 2014. Threat-Based Security Analysis for the Internet of Things. In 2014 International Workshop on Secure Internet of Things. 35-43. https://doi.org/10.1109/SIoT.2014.10

[4] Thijs Baars, Frederik Mijnhardt, Kevin Vlaanderen, and Marco Spruit. 2016. An Analytics Approach to Adaptive Maturity Models Using Organizational Characteristics. Decision Analytics 3, 1 (Nov. 2016), 5. https://doi.org/10.1186/s40165016-0022-1

[5] Matthew P. Barrett. 2018. Framework for Improving Critical Infrastructure Cybersecurity Version 1.1. Technical Report. NIST. 
[6] Mohamed Slim Ben Mahmoud, Nicolas Larrieu, and Alain Pirovano. 2011. A Risk Propagation Based Quantitative Assessment Methodology for Network Security Aeronautical Network Case Study. In 2011 Conference on Network and Information Systems Security. 1-9. https://doi.org/10.1109/SAR-SSI.2011.5931372

[7] Michael Benz and Dave Chatterjee. 2020. Calculated Risk? A Cybersecurity Evaluation Tool for SMEs. Business Horizons 63, 4 (July 2020), 531-540. https //doi.org/10.1016/j.bushor.2020.03.010

[8] J. F. Carías, M. R. S. Borges, L. Labaka, S. Arrizabalaga, and J. Hernantes. 2020 Systematic Approach to Cyber Resilience Operationalization in SMEs. IEEE Access 8 (2020), 174200-174221. https://doi.org/10.1109/ACCESS.2020.3026063

[9] Valentina Casola, Alessandra De Benedictis, Massimiliano Rak, and Umberto Villano. 2019. Toward the Automation of Threat Modeling and Risk Assessment in IoT Systems. Internet of Things 7 (Sept. 2019), 100056. https://doi.org/10.1016/ j.iot.2019.100056

[10] Valentina Casola, Alessandra De Benedictis, Massimiliano Rak, and Umberto Villano. 2020. A Novel Security-by-Design Methodology: Modeling and Assessing Security by SLAs with a Quantitative Approach. Fournal of Systems and Software 163 (May 2020), 110537. https://doi.org/10.1016/j.jss.2020.110537

[11] Louis Anthony Cox. 2008. Some Limitations of "Risk $=$ Threat $\times$ Vulnerability $\times$ Consequence" for Risk Analysis of Terrorist Attacks. Risk Analysis 28, 6 (2008), 1749-1761. https://doi.org/10.1111/j.1539-6924.2008.01142.x

[12] Matthew C. Davis, Rose Challenger, Dharshana N. W. Jayewardene, and Chris W. Clegg. 2014. Advancing Socio-Technical Systems Thinking: A Call for Bravery. Applied Ergonomics 45, 2, Part A (March 2014), 171-180. https://doi.org/10.1016/ j.apergo.2013.02.009

[13] Edward L. Deci and Richard M. Ryan. 1985. The General Causality Orientations Scale: Self-Determination in Personality. Fournal of Research in Personality 19, 2 (June 1985), 109-134. https://doi.org/10.1016/0092-6566(85)90023-6

[14] Mina Deng, Kim Wuyts, Riccardo Scandariato, Bart Preneel, and Wouter Joosen. 2011. A Privacy Threat Analysis Framework: Supporting the Elicitation and Fulfillment of Privacy Requirements. Requirements Engineering 16, 1 (March 2011), 3-32. https://doi.org/10.1007/s00766-010-0115-7

[15] ENISA. 2007. A Simplified Approach to Risk Management for SMEs. Report. ENISA

[16] ENISA. 2016. ENISA Threat Taxonomy. https://www.enisa.europa.eu/topics/threat-risk-management/threats-andtrends/enisa-threat-landscape/threat-taxonomy.

[17] ENISA. 2019. ENISA Threat Landscape Report 2018. Report. ENISA.

[18] ENISA. 2020. ENISA Threat Landscape 2020 - List of Top 15 Threats. Report. ENISA.

[19] European Commission. 2016. SME Definition. https://ec.europa.eu/growth/smes/sme-definition.

[20] European DIGITAL SME Alliance. 2020. The EU Cybersecurity Act and the Role of Standards for SMEs - Position Paper. Technical Report. Brussels.

[21] GEIGER Consortium. 2020. GEIGER Project Website. https://project.cybergeiger.eu/.

[22] D. Gollmann, C. Herley, V. Koenig, W. Pieters, and M. A. Sasse. 2015. SocioTechnical Security Metrics. Dagstuhl Reports, 4, 2015 (2015). https://doi.org/10. 4230/DagRep.4.12.1

[23] Bartlomiej Hanus and Yu "Andy" Wu. 2016. Impact of Users' Security Awareness on Desktop Security Behavior: A Protection Motivation Theory Perspective. Information Systems Management 33, 1 (Jan. 2016), 2-16. https://doi.org/10.1080/ 10580530.2015 .1117842

[24] Margareta Heidt, Jin P. Gerlach, and Peter Buxmann. 2019. Investigating the Security Divide between SME and Large Companies: How SME Characteristics Influence Organizational IT Security Investments. Information Systems Frontiers 21, 6 (Dec. 2019), 1285-1305. https://doi.org/10.1007/s10796-019-09959-1

[25] International Organization for Standardization (ISO)/International Electrotechnical Commission (IEC). 2012. ISO/IEC 27032:2012 Information Technology Security Techniques - Guidelines for Cybersecurity. Technical Report. ISO/IEC.

[26] International Organization for Standardization (ISO)/International Electrotechnical Commission (IEC). 2013. ISO/IEC 27002:2013 Information Technology Security Techniques - Code of Practice for Information Security Controls. Technical Report. ISO/IEC.

[27] Urpo Kaila and Linus Nyman. 2018. Information Security Best Practices: First Steps for Startups and SMEs. Technology Innovation Management Review 8, 11 (2018), 32-42. https://doi.org/10.22215/timreview/1198

[28] Younghwa Lee and Kai R. Larsen. 2009. Threat or Coping Appraisal: Determinants of SMB Executives' Decision to Adopt Anti-Malware Software. European fournal of Information Systems 18, 2 (April 2009), 177-187. https://doi.org/10.1057/ejis. 2009.11

[29] Yang Liu, Armin Sarabi, Jing Zhang, Parinaz Naghizadeh, Manish Karir, Michael Bailey, and Mingyan Liu. 2015. Cloudy with a Chance of Breach: Forecasting Cyber Security Incidents. In 24th \{USENIX\} Security Symposium (\{USENIX\} Security 15). $1009-1024$

[30] Louis Marinos and Andreas Sfakianakis. 2013. ENISA Threat Landscape 2012. Report. ENISA.

[31] Marijn Martens, Ralf De Wolf, and Lieven De Marez. 2019. Investigating and Comparing the Predictors of the Intention towards Taking Security Measures against
Malware, Scams and Cybercrime in General. Computers in Human Behavior 92 (March 2019), 139-150. https://doi.org/10.1016/j.chb.2018.11.002

[32] Philip Menard, Gregory J. Bott, and Robert E. Crossler. 2017. User Motivations in Protecting Information Security: Protection Motivation Theory Versus SelfDetermination Theory. Fournal of Management Information Systems 34, 4 (Oct. 2017), 1203-1230. https://doi.org/10.1080/07421222.2017.1394083

[33] Frederik Mijnhardt, Thijs Baars, and Marco Spruit. 2016. Organizational Characteristics Influencing SME Information Security Maturity. Fournal of Computer Information Systems 56, 2 (April 2016), 106-115. https://doi.org/10.1080/08874417. 2016.1117369

[34] Michael Muckin and Scott C Fitch. 2019. A Threat-Driven Approach to Cyber Security. Technical Report. Lockheed Martin Corporation. 45 pages.

[35] NCSC UK. 2014. Cyber Essentials. https://www.ncsc.gov.uk/cyberessentials/overview.

[36] Keshnee Padayachee. 2012. Taxonomy of Compliant Information Security Behavior. Computers \& Security 31, 5 (July 2012), 673-680. https://doi.org/10.1016/ j.cose.2012.04.004

[37] Charles P. Pfleeger and Shari Lawrence Pfleeger. 2012. Analyzing Computer Security: A Threat/Vulnerability/Countermeasure Approach. Prentice Hall Professional.

[38] Atle Refsdal, Bjørnar Solhaug, and Ketil Stolen. 2015. Cyber-Risk Management. Springer International Publishing.

[39] James Riordan and R. P. Lippmann. 2016. Threat-Based Risk Assessment for Enterprise Networks.

[40] Richard M. Ryan and Edward L. Deci. 2000. Self-Determination Theory and the Facilitation of Intrinsic Motivation, Social Development, and Well-Being. American Psychologist 55, 1 (2000), 68-78. https://doi.org/10.1037/0003-066X.55. 1.68

[41] Armin Sarabi, Parinaz Naghizadeh, Yang Liu, and Mingyan Liu. 2016. Risky Business: Fine-Grained Data Breach Prediction Using Business Profiles. fournal of Cybersecurity 2, 1 (Dec. 2016), 15-28. https://doi.org/10.1093/cybsec/tyw004

[42] Riccardo Scandariato, Kim Wuyts, and Wouter Joosen. 2015. A Descriptive Study of Microsoft's Threat Modeling Technique. Requirements Engineering 20, 2 (June 2015), 163-180. https://doi.org/10.1007/s00766-013-0195-2

[43] Stephan Schmidt and Sahin Albayrak. 2010. A Quantitative Framework for Dependency-Aware Organizational IT Risk Management. In 2010 10th International Conference on Intelligent Systems Design and Applications. 1207-1212. https://doi.org/10.1109/ISDA.2010.5687022

[44] Alireza Shameli-Sendi, Rouzbeh Aghababaei-Barzegar, and Mohamed Cheriet. 2016. Taxonomy of Information Security Risk Assessment (ISRA). Computers \& Security 57 (March 2016), 14-30. https://doi.org/10.1016/j.cose.2015.11.001

[45] Alireza Shojaifar and Samuel A. Fricker. 2020. SMEs' Confidentiality Concerns for Security Information Sharing. In Human Aspects of Information Security and Assurance (IFIP Advances in Information and Communication Technology), Nathan Clarke and Steven Furnell (Eds.). Springer International Publishing, Cham, 289299. https://doi.org/10.1007/978-3-030-57404-8_22

[46] Alireza Shojaifar, Samuel A. Fricker, and Martin Gwerder. 2020. Automating the Communication of Cybersecurity Knowledge: Multi-Case Study. In Information Security Education. Information Security in Action (IFIP Advances in Information and Communication Technology), Lynette Drevin, Suné Von Solms, and Marianthi Theocharidou (Eds.). Springer International Publishing, Cham, 110-124. https: //doi.org/10.1007/978-3-030-59291-2_8

[47] Marco Spruit and Martijn Roeling. 2014. ISFAM: The Information Security Focus Area Maturity Model. ECIS 2014 Proceedings (June 2014).

[48] G. Stergiopoulos, D. Gritzalis, and V. Kouktzoglou. 2018. Using Formal Distributions for Threat Likelihood Estimation in Cloud-Enabled IT Risk Assessment. Computer Networks 134 (April 2018), 23-45. https://doi.org/10.1016/j.comnet. 2018.01.033

[49] Swiss NCSC. 2021. Cyberthreats. https://www.ncsc.admin.ch/ncsc/en/home.html.

[50] Brett Tucker. 2020. Advancing Risk Management Capability Using the OCTAVE FORTE Process. (Dec. 2020). https://doi.org/10.1184/R1/13014266.v1

[51] Robert J. Vallerand. 1997. Toward A Hierarchical Model of Intrinsic and Extrinsic Motivation. In Advances in Experimental Social Psychology, Mark P. Zanna (Ed.). Vol. 29. Academic Press, 271-360. https://doi.org/10.1016/S0065-2601(08)60019-2

[52] René van Bavel, Nuria Rodríguez-Priego, José Vila, and Pam Briggs. 2019. Using Protection Motivation Theory in the Design of Nudges to Improve Online Security Behavior. International fournal of Human-Computer Studies 123 (March 2019), 29-39. https://doi.org/10.1016/j.ijhcs.2018.11.003

[53] Kim Wuyts, Riccardo Scandariato, and Wouter Joosen. 2014. Empirical Evaluation of a Privacy-Focused Threat Modeling Methodology. Fournal of Systems and Software 96 (Oct. 2014), 122-138. https://doi.org/10.1016/j.jss.2014.05.075

[54] Wenjun Xiong and Robert Lagerström. 2019. Threat Modeling - A Systematic Literature Review. Computers \& Security 84 (July 2019), 53-69. https://doi.org/ 10.1016/j.cose.2019.03.010

[55] Bilge Yigit Ozkan and Marco Spruit. 2020. Addressing SME Characteristics for Designing Information Security Maturity Models. In Human Aspects of Information Security and Assurance (IFIP Advances in Information and Communication 
Technology), Nathan Clarke and Steven Furnell (Eds.). Springer International Publishing, Cham, 161-174. https://doi.org/10.1007/978-3-030-57404-8_13

[56] Bilge Yigit Ozkan, Marco Spruit, Roland Wondolleck, and Verónica Burriel Coll. 2019. Modelling Adaptive Information Security for SMEs in a Cluster. Fournal of Intellectual Capital 21, 2 (Jan. 2019), 235-256. https://doi.org/10.1108/JIC-05-
2019-0128

[57] Bilge Yigit Ozkan, Sonny van Lingen, and Marco Spruit. 2021. The Cybersecurity Focus Area Maturity (CYSFAM) Model. Journal of Cybersecurity and Privacy 1,1 (March 2021), 119-139. https://doi.org/10.3390/jcp1010007 